\title{
Density gradient centrifugation and swim-up for ICSI: useful, unsafe, or just unsuitable?
}

\author{
Hamilton De Martin ${ }^{1,2,3} \cdot$ Eduardo P. Miranda $^{4}$ (D) $\cdot$ Marcello S. Cocuzza ${ }^{1,5} \cdot$ Pedro A. A. Monteleone ${ }^{1,2,3}$
}

Received: 21 September 2019 / Accepted: 9 October 2019 / Published online: 29 October 2019

(C) Springer Science+Business Media, LLC, part of Springer Nature 2019

Intracytoplasmic sperm injection (ICSI) revolutionized the treatment of male factor infertility [1]. ICSI eliminates all natural selection barriers, since the spermatozoon is directly injected into the oocyte cytoplasm [2]. While visual inspection permits a trained embryologist to identify immature spermatozoa, the damage in sperm DNA itself does not result in any morphological changes, making its identification nearly impossible [3]. Therefore, semen processing prior to ICSI procedures is of utmost importance.

Density gradient centrifugation (DGC) and swim-up are among the most used sperm selection techniques in clinical practice $[4,5]$. A recent study by Muratori et al. has evaluated the efficacy of both techniques in removing DNA-damaged spermatozoa and reported alarming results [6]. Using a modified TUNEL (terminal deoxynucleotidyl transferase (TdT)mediated fluorescein-dUTP nick end labeling) technique in which viable and nonviable DNA-damaged spermatozoa could be identified (live TUNEL), the authors suggested that among sub/infertile men, there is a fraction of subjects experiencing an increase of sperm DNA damage during selection with DGC and swim-up [6]. In this analysis, baseline seminal parameters were not able to predict increased or decreased DNA damage after processing. While the authors have acknowledged that semen processing procedures

Eduardo P. Miranda

mirandaedp@gmail.com

1 Human Reproduction Center, Sao Paulo, Brazil

2 Discipline of Gynecology, Hospital das Clinicas, University of Sao Paulo Medical School, Sao Paulo, Brazil

3 Monteleone Clinic, Sao Paulo, Brazil

4 Division of Urology, Universidade Federal do Ceara, Fortaleza, Brazil

5 Division of Urology, Hospital das Clinicas, University of Sao Paulo Medical School, Sao Paulo, Brazil increase DNA fragmentation in some subjects and decrease in others, no plausible scientific explanation was given.

This article has provided relevant information to the field of reproductive medicine. However, we intend to discuss the possible mechanisms involved in this phenomenon based on numerous clinical and experimental studies available. Didactically, sperm with damaged DNA could be divided into two categories: immature spermatozoa and senescent mature spermatozoa. Immature spermatozoa have poorly packed chromatin as a result of incomplete protamination, which makes them vulnerable to reactive oxygen species (ROS) attack [7]. On the other hand, although senescent mature sperm have compact chromatin and normal morphology, DNA damage might be a result of mitochondrial impairment following an apoptotic cascade [8]. These mature and senescent groups could be further divided into different subcategories: those in an incipient apoptotic process with moderate mitochondrial and DNA impairment and those with a more pronounced mitochondrial and DNA impairment, plasma membrane damage, and externalization of phosphatidylserine.

Both DCG and swim-up methodologies are intended to isolate viable sperm. DCG tends to concentrate motile spermatozoa in bottom layers while seminal plasma, debris, round cells, dead sperm, and immature sperm are retained in the upper layers. Interestingly, one may suggest that DGC functions similarly to the cervical mucus in sorting sperm with higher density. Spermatozoa with compact chromatin and at least reasonable motility can reach the bottom of the conical tube even in the presence of mitochondrial impairment and DNA damage. Therefore, DCG can successfully retain only immature forms, concentrating mature and morphologically normal forms, which might increase the risk of choosing a DNA-fragmented sperm at the time of ICSI.

According to our hypotheses, if a semen sample has a large population of affected immature sperm, there will be a reduction in the sperm DNA fragmentation rate (SDF) by retaining them in the upper layers of the gradient. In contrast, if many affected mature sperm are present, there will be an increase in 
SDF, not only by concentrating the affected mature forms but also by increasing the damage of incipient forms (possibly by ROS attack), making them detectable by TUNEL.

Although the role of mitochondria in sperm function is still a matter of debate, several studies report that sperm motility is closely related to mitochondrial membrane potential, which is also associated with DNA integrity $[9,10]$. Therefore, we may suggest that spermatozoa capable of sustained migratory efforts should have an intact DNA. Nature takes advantage of this fact efficiently by imposing obstacles that challenge the functionality of mitochondria and ultimately assuring chromatin integrity. Even though the swim-up procedure uses motility to select more functional sperm, it has noticeable limitations. Not only would it not retain motile immature spermatozoa, but it would also allow sperm in an incipient apoptotic state to be able to move into the collection area.

Therefore, it is our opinion that both DCG and swim-up procedures are not the best semen processing strategies for ICSI. It is not our understanding that these procedures cause damage themselves, but they are not effective in selecting sperm without DNA damage in a consistent fashion, especially because they were not designed for this purpose. In this scenario, an ideal method for sperm selection is yet to be determined. It is desirable that it should be able to replicate similar principles found in the female genital tract.

Comparing spermatozoa migration through channels of different lengths, Tasoglu et al. [11] determined that a $2-\mathrm{cm}$ migration is enough to obtain functional human spermatozoa, as sperm exhaustion has been considered an important principle in microfluidic sperm selection. In this scenario, a microfluidic device for clinical use was developed based on experimental data [12]. Briefly, the spermatozoa must actively swim from the inlet reservoir through the main channel to the outlet reservoir to be collected. Despite the absence of flow in the circuit, a considerable migratory effort was still required from the most motile spermatozoa. Those with low resistance to migration would not reach the collection area and would therefore be eliminated. Two recently published studies have found that the spermatozoa recovered with this type of device exhibit greater vitality, better morphology, and less DNA damage compared with DGC and swim-up [13,14]. Nosrati et al. [15] developed a different device consisting in a radial arrangement of 500 channels filled with a viscoelastic medium without any flow mechanism. However, the viscosity of the surrounding medium makes the migration even more challenging. The authors have demonstrated that processing of raw semen samples in such device yields an improvement of $89 \%$ in sperm vitality and $80 \%$ in DNA integrity. De Martin et al. [16] tested an integrated system composed of long-distance, countercurrent, and viscous media migration. In this inverted flow system, there is a difference of hydrostatic pressure between the reservoirs, generating a flow of liquid from the outlet reservoir toward the inlet reservoir. In addition, polyvinylpyrrolidone (PVP) is inserted in the distal end of the outlet reservoir, which generates a viscosity gradient. Spermatozoa that are able to reach the distal end of the outlet reservoir may be collected with an ICSI needle and injected in the oocyte. This device was able to reduce uncondensed chromatin of spermatozoa to nearly $1 \%$ also using raw semen samples. Likewise, Wu et al. [17] described the flowing upstream sperm sorting (FUSS). According to microfluidic principles applied to this device, the spermatozoa initially deposited in the inlet are drawn out by a strong flow toward the outlet at the first segment of the circuit (straight-flow zone). The channel has a dilated intermediate segment (diffuser-type sperm sorter) that slows down the flow, allowing the spermatozoa to swim against the fluid stream (positive rheotaxis). This system was able to recover enriched samples containing $80 \%$ of viable sperm in up to $15 \mathrm{~min}$ after processing semen samples of $\sim 200,000$ spermatozoa.

Perhaps these methodologies could add a more natural selection process to ICSI, thus mitigating the theoretical risks involved in this procedure. Although more studies are necessary to confirm preliminary findings, these novel selection methodologies apparently submit sperm populations to a more robust selection pressure, which could potentially reduce SDF in assisted reproductive techniques.

\section{References}

1. Esteves SC, Roque M, Bedoschi G, Haahr T, Humaidan P. Intracytoplasmic sperm injection for male infertility and consequences for offspring. Nat Rev Urol. 2018;15(9):535-62.

2. Palermo G, Joris H, Devroey P, Van Steirteghem AC. Pregnancies after intracytoplasmic injection of single spermatozoon into an oocyte. Lancet. 1992;340(8810):17-8.

3. Avendaño C, Franchi A, Taylor S, Morshedi M, Bocca S, Oehninger S. Fragmentation of DNA in morphologically normal human spermatozoa. Fertil Steril. 2009;91(4):1077-84.

4. Henkel R. Sperm preparation: state-of-the-art-physiological aspects and application of advanced sperm preparation methods. Asian J Androl. 2012;14(2):260-9.

5. Rappa KL, Rodriguez HF, Hakkarainen GC, Anchan RM, Mutter GL, Asghar W. Sperm processing for advanced reproductive technologies: where are we today? Biotechnol Adv. 2016;34(5):57887.

6. Muratori M, Tarozzi N, Carpentiero F, Danti S, Perrone FM, Cambi $\mathrm{M}$, et al. Sperm selection with density gradient centrifugation and swim up: effect on DNA fragmentation in viable spermatozoa. Sci Rep. 2019;9(1):7492.

7. Carrell DT, Emery BR, Hammoud S. Altered protamine expression and diminished spermatogenesis: what is the link? Hum Reprod Update. 2007;13(3):313-27.

8. Koppers AJ, Mitchell LA, Wang P, Lin M, Aitken RJ. Phosphoinositide 3-kinase signalling pathway involvement in a truncated apoptotic cascade associated with motility loss and oxidative DNA damage in human spermatozoa. Biochem J. 2011;436(3):687-98.

9. Moscatelli N, Spagnolo B, Pisanello M, Lemma ED, De Vittorio M, Zara V, et al. Single-cell-based evaluation of sperm progressive 
motility via fluorescent assessment of mitochondria membrane potential. Sci Rep. 2017;7(1):17931.

10. Amaral S, Tavares R, Baptista M, Sousa MI, Silva A, EscadaRebelo S, et al. Mitochondrial functionality and chemical compound action on sperm function. Curr Med Chem. 2016;23(31): 3575-606.

11. Tasoglu S, Safaee H, Zhang X, Kingsley JL, Catalano PN, Gurkan $\mathrm{UA}$, et al. Exhaustion of racing sperm in nature-mimicking microfluidic channels during sorting. Small. 2013;9(20):3374-84.

12. Chinnasamy T, Kingsley JL, Inci F, Turek PJ, Rosen MP, Behr B, et al. Guidance and self-sorting of active swimmers: 3D periodic arrays increase persistence length of human sperm selecting for the fittest. Adv Sci (Weinh). 2018;5(2):1700531.

13. Quinn MM, Jalalian L, Ribeiro S, Ona K, Demirci U, Cedars MI, et al. Microfluidic sorting selects sperm for clinical use with reduced DNA damage compared to density gradient centrifugation with swim-up in split semen samples. Hum Reprod. 2018.
14. Parrella A, Keating D, Cheung S, Xie P, Stewart JD, Rosenwaks Z, et al. A treatment approach for couples with disrupted sperm DNA integrity and recurrent ART failure. J Assist Reprod Genet. 2019.

15. Nosrati R, Vollmer M, Eamer L, San Gabriel MC, Zeidan K, Zini A, et al. Rapid selection of sperm with high DNA integrity. Lab Chip. 2014;14(6):1142-50.

16. De Martin H, Cocuzza MS, Tiseo BC, Wood GJA, Miranda EP, Monteleone PAA, et al. Positive rheotaxis extended drop: a onestep procedure to select and recover sperm with mature chromatin for intracytoplasmic sperm injection. J Assist Reprod Genet. 2017;34(12):1699-708.

17. Wu JK, Chen PC, Lin YN, Wang CW, Pan LC, Tseng FG. Highthroughput flowing upstream sperm sorting in a retarding flow field for human semen analysis. Analyst. 2017;142(6):938-44.

Publisher's note Springer Nature remains neutral with regard to jurisdictional claims in published maps and institutional affiliations. 